\title{
ON THE CHARACTERS OF A SEMISIMPLE LIE GROUP
}

\section{HARISH-CHANDRA}

Let $G$ be a connected semisimple Lie group and let $Z$ denote its center. If $\pi$ is a representation [2c] of $G$ on a Hilbert space $\mathfrak{S}$ we consider the space $V$ consisting of all finite linear combinations of elements of the form

$$
\int f(x) \pi(x) \psi d x \quad\left(f \in C_{c}^{\infty}(G), \psi \in \mathfrak{W}\right),
$$

where $d x$ is the Haar measure of $G$ and $C_{c}^{\infty}(G)$ is the set of all (complexvalued) functions on $G$ which are everywhere indefinitely differentiable and which vanish outside a compact set. $V$ is called the Gårding subspace of $\mathfrak{S}$. Let $R$ and $C$ be the fields of real and complex numbers respectively and $g_{0}$ the Lie algebra of $G$. We complexify $g_{0}$ to $\mathfrak{g}$ and denote by $\mathfrak{B}$ the universal enveloping algebra of $\mathfrak{g}[2 \mathfrak{a}]$. Then there exists a (uniquely determined) representation $\pi_{V}$ of $\mathfrak{B}$ on $V$ such that $\pi_{V}(X) \psi=\lim _{t \rightarrow 0}(1 / t)\{\pi(\exp t X) \psi-\psi\} \quad\left(X \in \mathfrak{g}_{0}, \psi \in V, t \in R\right)$. Let 3 denote the center of $\mathfrak{B}$. We say that $\pi$ is quasi-simple if there exist homomorphisms $\eta$ and $\chi$ of $Z$ and 8 respectively into $C$ such that $\pi(\zeta) \phi=\eta(\zeta) \phi, \pi_{V}(z) \psi=\chi(z) \psi$ for all $\zeta \in Z, z \in \mathcal{Z}, \phi \in \mathfrak{S}$ and $\psi \in V . \eta$ is then called the central character and $\chi$ the infinitesimal character of $\pi$. An irreducible unitary representation is automatically quasi-simple [5].

Let $A$ be a bounded linear operator on $\mathfrak{S}$. We say that $A$ is of the trace class or $A$ has a trace if for every complete orthonormal set $\left(\psi_{j}\right)_{j \in J}$ in $\mathfrak{S}$ the series ${ }^{1} \sum_{j \in J}\left(\psi_{j}, A \psi_{j}\right)$ converges absolutely and its sum is independent of the choice of the complete orthonormal set. ${ }^{2}$ We call this sum the trace of $A$ and denote it by $\operatorname{Sp} A$. Now suppose $\pi$ is quasi-simple and irreducible. Then it can be shown (see [2e]) that for any $f \in C_{c}^{\infty}(G)$ the operator $\int f(x) \pi(x) d x$ is of the trace class. If we denote its trace by $T_{\pi}(f)$ we get a linear function $T_{\pi}$ on $C_{c}^{\infty}(G)$ which is actually a distribution (see [4; and $2 \mathrm{e}]$ ). We call this distribution the character of $\pi$. Our object is to try to determine $T_{\pi}$.

An address delivered before the New York meeting of the Society on February 25, 1955 by invitation of the Committee to Select Hour Speakers for Eastern Sectional Meetings; received by the editors March 28, 1955.

${ }^{1}$ As usual $(\phi, \psi)$ denotes the scalar product of the two elements $\phi$ and $\psi$ in $\mathfrak{S}$.

2 Actually it can be shown that this independence of the sum follows automatically from the absolute convergence of the series for every orthonormal base. 
Let $x \rightarrow \operatorname{Ad}(x)(x \in G)$ denote the adjoint representation of $G$. If $\lambda$ is an indeterminate and $I$ is the identity mapping of $\mathfrak{g}_{0}$ we consider the characteristic polynomial $P_{x}(\lambda)=\operatorname{det}(\lambda I-\operatorname{Ad}(x))$ of $\operatorname{Ad}(x)$. Let $l$ be the highest integer such that $(\lambda-1)^{l}$ divides $P_{x}(\lambda)$ for every $x \in G$. We expand $P_{x}(\lambda)$ in powers of $(\lambda-1)$ and denote by $D(x)$ the coefficient of $(\lambda-1)^{l}$ in this expansion. Then it is clear that $D$ is an analytic function on $G$ which, in view of our definition of $l$, cannot be identically zero. The integer $l$ is called the rank of $G$. Let $S$ denote the set of all $x \in G$ for which $D(x)=0$. Then $S$ is a closed, nowhere dense subset of $G$ and its complement $G^{\prime}$ is open and everywhere dense in $G$. An element $x \in G$ is called singular or regular according as $x \in S$ or $x \in G^{\prime}$. Since Ad $(z x)=\operatorname{Ad}(x)(z \in Z)$ it is obvious that $Z S=S$ and $Z G^{\prime}=G^{\prime}$. Also since $P_{y x y^{-1}}(\lambda)=P_{x}(\lambda) \quad(x, y \in G)$, it follows that $D\left(y x y^{-1}\right)=D(x)$ and therefore $y S y^{-1}=S, y G^{\prime} y^{-1}=G^{\prime}(y \in G)$.

When speaking of a real differentiable (or analytic) manifold $M$ let us agree to include the case when $M$ is not connected but the various connected components of $M$, which are all manifolds in the usual sense, have the same dimension. Under this definition every open subset $U$ of $M$ is again a manifold. Let $C_{c}^{\infty}(U)$ denote the set of all complex-valued functions on $M$ which are indefinitely differentiable and which vanish outside some compact subset of $U$. In particular suppose $U$ is an open subset of $G$ and $F$ is a (complex-valued) function on $U$. We say that $F$ is locally summable if it is summable on every compact subset of $U$ with respect to the Haar measure of $G$. $T$ being a distribution on $G$ we say that $T=F$ on $U$ if $F$ is locally summable and

$$
T(f)=\int f(x) F(x) d x
$$

for all $f \in C_{c}^{\infty}(U)$. Our main result may now be stated as follows.

THEOREM 1. Let $\pi$ be an irreducible quasi-simple representation of $G$ on $\mathfrak{S}$ and let $T_{\pi}$ denote its character. Then there exists an analytic function $F_{\pi}$ on $G^{\prime}$ such that $T_{\pi}=F_{\pi}$ on $G^{\prime}$.

Although in general $G^{\prime}$ is not connected, there always exist a finite number of connected components $G_{1}, \cdots, G_{r}$ of $G^{\prime}$ such that $Z G_{i}$ $\cap Z G_{j}=\varnothing$ if $i \neq j$ and $G^{\prime}=\bigcup_{i=1}^{r} Z G_{i}$. Moreover if $\eta_{\pi}$ is the central character of $\pi$ it is easy to show that $F_{\pi}(z x)=\eta_{\pi}(z) F_{\pi}(x)\left(z \in Z, x \in G^{\prime}\right)$. Hence the knowledge of $F_{\pi}$ on $G_{1} \cup G_{2} \cup \ldots \cup G_{r}$ is sufficient to determine it completely. On the other hand it is possible to give examples in which $F_{\pi}$ vanishes everywhere on one of the components $G_{i}$ without being zero identically on $G^{\prime}$. However in case $G$ is either a 
compact or a complex group, $G^{\prime}$ is connected and then $F_{\pi}$ is completely determined by its restriction on any nonempty open subset of $G^{\prime}$.

For any $x \in G$ and $f \in C_{c}^{\infty}(G)$ we define the function $f^{x}$ by $f^{x}(y)$ $=f\left(x^{-1} y x\right) \quad(y \in G)$. Then $f^{x} \in C_{c}^{\infty}(G)$ and $T_{\pi}\left(f^{x}\right)=T_{\pi}(f)$ (see [2e]). From this it follows that $F_{\pi}\left(x y x^{-1}\right)=F_{\pi}(y)\left(x \in G, y \in G^{\prime}\right)$. Now let $A$ be a maximal abelian subgroup of $G$ which is not contained in $S$. (We call such a group a Cartan subgroup of $G$.) Obviously $A$ is closed in $G$. Let $A^{\prime}=A \cap G^{\prime}$ and $V=\bigcup_{x \in G} x A^{\prime} x^{-1}$. Then $V$ is an open subset of $G^{\prime}$ and it is clear from the above remarks that $F_{\pi}$ is determined completely on $V$ as soon as we know it on $A^{\prime}$. Let $\mathfrak{h}_{0}$ denote the subalgebra of $\mathfrak{g}_{0}$ corresponding to $A$. Then the complexification $\mathfrak{h}$ of $\mathfrak{h}_{0}$ is a Cartan subalgebra of $\mathfrak{g}$. Let $W$ be the Weyl group (see $[\mathbf{2 b}]$ ) of $\mathfrak{g}$ with respect to $\mathfrak{h}$ so that $W$ is a finite group of nonsingular linear transformations of $\mathfrak{h}$. If $\Lambda$ is a linear function on $\mathfrak{h}$ and $s \in W$ we define the linear function $s \Lambda$ by the rule $s \Lambda(H)=\Lambda\left(s^{-1} H\right)(H \in \mathfrak{h})$. Let $\left(H_{1}, \cdots, H_{l}\right)$ be a base for $\mathfrak{h}$ over $C$. A function $P$ on $\mathfrak{h}$ is called a polynomial function if there exists a polynomial $p\left(x_{1}, \cdots, x_{l}\right)$ in $l$ variables $\left(x_{1}, \cdots, x_{l}\right)$ with coefficients in $C$ such that $P(H)=p\left(a_{1}, \cdots, a_{l}\right)$ if $H=a_{1} H_{1}$ $+\cdots+a_{l} H_{l}\left(a_{i} \in C\right)$. The degree of $p$ is also called the degree of $P$. Clearly these definitions are independent of the choice of the base $\left(H_{1}, \cdots, H_{l}\right)$.

THEOREM 2. Let $\pi$ and $F_{\pi}$ be as in Theorem 1. Then there exists a linear function $\Lambda$ on $\mathfrak{h}$ with the following property. For any $a \in A^{\prime}$ we can choose polynomial functions $p_{s}(s \in W)$ on $\mathfrak{h}$ such that

$$
F_{x}(a \exp H)=|D(a \exp H)|^{-1 / 2} \sum_{s \in W} p_{s}(H) e^{s \Lambda(H)}
$$

for all $H$ lying sufficiently near zero in $\mathfrak{h}_{0} . \Lambda$ is unique up to an operation of $W$ and if $N$ is the number of elements $\sigma$ in $W$ such that $\Lambda=\sigma \Lambda$, the degree of every $p_{s}$ is necessarily smaller than $N$.

In particular if $s \Lambda \neq \Lambda$ except when $s$ is the unit element of $W, p_{s}$ must all be constants. (It is hardly necessary to point out the resemblance of the above formula to the one given by Weyl [6] for the irreducible characters of a compact semisimple Lie group. It should also be compared with the results of Gelfand and Naimark [7] on the unitary characters of the complex classical groups (see also [2f, p. 511])). Although $A^{\prime}$ is not necessarily connected, it is possible to select a finite set $B_{1}, \cdots, B_{r}$ of its connected components such that $A^{\prime}=\mathrm{U}_{i=1}^{r} Z B_{i}$. Therefore in order to determine $F_{\pi}$ on $A^{\prime}$, it is sufficient to know $\eta_{\pi}$ and the restrictions of $F_{\pi}$ on some nonempty open subsets 
of $B_{1}, \cdots, B_{r}$. Hence if $\Lambda$ is known, Theorem 2 gives us a formula for $F_{\pi}$ on $A^{\prime}$ in terms of a finite number of undetermined constants. On the other hand we shall see presently that $\Lambda$ is completely determined (up to an operation of $W$ ) by the infinitesimal character $\chi_{\pi}$ of $\pi$.

Two Cartan subgroups $A_{1}$ and $A_{2}$ are said to be conjugate if $A_{2}=x A_{1} x^{-1}$ for some $x \in G$. It is always possible to choose a finite number of distinct Cartan subgroups $A_{1}, \cdots, A_{k}$ such that every Cartan subgroup is conjugate to exactly one of these. Then if $A_{i}^{\prime}=A_{i} \cap G^{\prime}$ and $V_{i}=\mathrm{U}_{x \in G} x A_{i}^{\prime} x^{-1}, G^{\prime}$ is the disjoint union of $V_{1}, \cdots, V_{k}$. This shows that if $\eta_{\pi}$ and $\chi_{\pi}$ are known, $F_{\pi}$ is completely determined in terms of a finite number of constants. ${ }^{3}$

Now we come to a brief outline of the proof. Let $M$ be a differentiable manifold, $Q$ a linear mapping of $C_{c}^{\infty}(M)$ into itself and $x_{0}$ a point in $M$. We say that $Q$ is a differential operator at $x_{0}$ if there exists a coordinate system $\left(t_{1}, \cdots, t_{m}\right)$ valid on an open neighborhood $U$ of $x_{0}$ and indefinitely differentiable functions $g_{i_{1} i_{2}} \cdots i_{p}$ on $U\left(1 \leqq i_{1}, \cdots\right.$, $\left.i_{p} \leqq m, 0 \leqq p \leqq q\right)$ such that if $f \in C_{c}^{\infty}(U), Q f$ is zero outside $U$ and

$$
Q f=\sum_{0 \leqq p \leqq q} \sum_{1 \leqq i_{1}, \cdots, i_{p} \leqq m} g_{i_{1} i_{2}} \cdots i_{i_{p}} \frac{\partial^{p}}{\partial t_{i_{1}} \cdots \partial t_{i_{p}}} f
$$

on $U$. If $Q$ is a differential operator at every point in $M$ we say simply that it is a differential operator (on $M$ ). $T$ being a distribution on $M$ and $Q$ a differential operator we define a distribution $Q^{\prime} T$ as follows:

$$
\left(Q^{\prime} T\right)(f)=T(Q f) \quad\left(f \in C_{c}^{\infty}(M)\right) .
$$

In particular if $g$ is an indefinitely differentiable function on $M$ it defines a differential operator $Q: f \rightarrow g f\left(f \in C_{c}^{\infty}(M)\right)$. In this case we write $g T$ to denote $Q^{\prime} T$ so that $(g T)(f)=T(g f)$. It is clear that the product of two differential operators is again a differential operator and therefore the differential operators form an algebra.

Coming back to $G$, we note that every $X \in \mathfrak{g}_{0}$ may be regarded as a differential operator on $G$ as follows:

$$
(X f)(x)=\left\{\frac{d}{d t} f(x \exp t X)\right\}_{t=0} \quad\left(f \in C_{c}^{\infty}(G), x \in G, t \in R\right) .
$$

Thus it is easy to see that $\mathfrak{B}$ may be identified in a natural way with a subalgebra of the algebra of differential operators on $G$. Then for any $b \in \mathfrak{B}$ we have a linear transformation $b^{\prime}$ of the space of distribu-

${ }^{3}$ Actually it is possible to improve Theorems 1 and 2 and show that $T_{\pi}$ coincides with an analytic function on an open subset of $G$ which, in general, is larger than $G^{\prime}$ and therefore has fewer connected components. 
tions on $G$. Since $\left(b_{1} b_{2}\right)^{\prime}=b_{2} b_{1}\left(b_{1}, b_{2} \in \mathfrak{B}\right)$ the mapping $b \rightarrow b^{\prime}$ is an antirepresentation of $\mathfrak{B}$. Let $\phi$ denote the anti-automorphism of $\mathfrak{B}$ over $C$ which is uniquely determined by the condition that $\phi(X)=-X$ $(X \in \mathfrak{g})$. Then $b \rightarrow(\phi(b))^{\prime}(b \in \mathfrak{B})$ is a representation of $\mathfrak{B} . T$ being any distribution on $G$ we now define $b T=(\phi(b))^{\prime} T(b \in \mathfrak{B})$. Then $(b T)(f)=T(\phi(b) f)\left(f \in C_{c}^{\infty}(G)\right)$. If $\chi_{\pi}$ is the infinitesimal character of $\pi$ and $z \in 3, f \in C_{c}^{\infty}(G)$, it is easy to see that

$$
\begin{aligned}
\int(\phi(z) f)(x) \pi(x) \psi d x & =\pi_{0}(z)\left(\int f(x) \pi(x) \psi d x\right) \\
& =\chi_{\pi}(z) \int f(x) \pi(x) \psi d x
\end{aligned}
$$

for all $\psi \in \mathfrak{S}$. (Here $\pi_{0}$ is the representation of $\mathfrak{B}$ on the Gårding subspace of $\mathfrak{S}$.) From this it follows that $z T_{\pi}=\chi_{\pi}(z) T_{\pi}$ for all $z \in \mathbb{Z}$. Hence $T_{\pi}$ is an eigen-distribution for each differential operator in $\mathbf{3}$.

On the other hand let $A$ be a Cartan subgroup of $G$. Put $A^{\prime}=A \cap G^{\prime}$ and $V=\mathrm{U}_{x \in G} x A^{\prime} x^{-1}$ as before. We regard $A^{\prime}$ and $V$ as open submanifolds of $A$ and $G$ respectively. Let $G^{*}$ be the factor space $G / A$ consisting of cosets of the form $x A(x \in G)$. If $h \in A$ and $x^{*} \in G^{*}$ we define $h^{x^{*}}=x h x^{-1}$ where $x$ is any element in the coset $x^{*}$. Let $d h$ and $d x^{*}$ respectively denote the Haar measure on $A$ and the invariant measure on $G^{*}$. Then we have the following lemma.

Lemma 1. There exists a distribution $\tau_{\pi}$ on $A^{\prime}$ with the following property. If $f \in C_{c}^{\infty}(V), T_{\pi}(f)=\tau_{\pi}(g)$ where $g$ is the function in $C_{c}^{\infty}\left(A^{\prime}\right)$ given by

$$
g(h)=|D(h)| \int_{G^{*}} f\left(h^{x *}\right) d x^{*} \quad\left(h \in A^{\prime}\right) .
$$

Let $\mathfrak{h}_{0}$ be the Lie algebra of $A$. Any element $H \in \mathfrak{h}_{0}$ may be regarded as a differential operator on $A$ so that if $g \in C_{0}^{\infty}(A)$,

$$
(H g)(h)=\left\{\frac{d}{d t} g(h \exp t H)\right\}_{t=0} \quad(h \in A, t \in R) .
$$

Let $\mathfrak{h}$ be the subspace of $\mathfrak{g}$ spanned by $\mathfrak{h}_{0}$ over $C$ and $\mathfrak{U}$ the subalgebra of $\mathfrak{B}$ generated by $(1, \mathfrak{h})$. Then $\mathfrak{U}$ may be identified in a natural way with a subalgebra of the algebra of differential operators on $A$. For any distribution $\tau$ on $A^{\prime}$ and $u \in \mathfrak{U}$ we define a distribution $u \tau$ on $A^{\prime}$ as follows:

$$
(u \tau)(g)=\tau(\phi(u) g)
$$$$
\left(g \in C_{c}^{\infty}\left(A^{\prime}\right)\right) .
$$

Here $\phi$ is the automorphism of $\mathfrak{u}$ given by $\phi(H)=-H(H \in \mathfrak{h})$. 
For every root $\alpha$ of $\mathfrak{g}$ (with respect to $\mathfrak{h}$ ), choose an element $X_{\alpha} \neq 0$ in $\mathfrak{g}$ such that $\left[H, X_{\alpha}\right]=\alpha(H) X_{\alpha}$ for all $H \in \mathfrak{h}$. We introduce some lexicographic order (see $[2 b]$ ) in the set of all roots and denote by $P$ the set of positive roots under this order. Put $\mathfrak{n}=\sum_{\alpha \in P} C X_{\alpha}$. Then for every $z \in \Omega$ there exists a unique element $\gamma^{\prime}(z) \in \mathfrak{U}$ such that $z-\gamma^{\prime}(z) \in \mathfrak{B n}($ see $[2 \mathrm{~b}$, p. 72$])$. If $2 \rho=\sum_{\alpha \in P} \alpha$ there exists a unique automorphism $\lambda$ of $\mathfrak{U}$ such that $\lambda(1)=1$ and $\lambda(H)=H-\rho(H)$ $(H \in \mathfrak{h})$. We put $\gamma(z)=\lambda\left(\gamma^{\prime}(z)\right)(z \in \mathbb{Z})$. Let $W$ be the Weyl group of $\mathfrak{g}$ with respect to $\mathfrak{h}$. It is clear that every $s \in W$ defines an automorphism $u \rightarrow u^{s}$ of $\mathfrak{U}$ such that $1^{s}=1$ and $H^{s}=s H(H \in \mathfrak{h})$. An element $u \in \mathfrak{U}$ is called an invariant if $u=u^{s}$ for all $s \in W$. Let $J$ be the subalgebra of $\mathfrak{U}$ consisting of all invariants. Then (see [2b, Lemma 38]) the mapping $z \rightarrow \gamma(z)(z \in ß)$ defines an isomorphism of $\rightrightarrows$ onto $J$. Now let $\Lambda$ be a linear function on $\mathfrak{h}$. We can extend it uniquely to a homomorphism of $\mathfrak{u}$ into $C$ which takes the value 1 at 1 . We agree to denote this extension also by $\Lambda$. Then as shown in [2b, Theorem 5] we can choose $\Lambda$ in such a way that $\chi_{\pi}(z)=\Lambda(\gamma(z))$ for all $z \in \mathbb{B}$. $\Lambda$ is determined up to an operation of $W$ by this condition.

Let $\Delta(h)=|D(h)|^{1 / 2}\left(h \in A^{\prime}\right)$. Then $\Delta$ is an analytic function on $A^{\prime}$ and therefore $\sigma_{\pi}=\Delta \tau_{\pi}$ is a well-defined distribution on $A^{\prime}$. Now if we transform the differential equation $z T_{\pi}=\chi_{\pi}(z) T_{\pi}(z \in \Re)$ for $T_{\pi}$ into a differential equation for $\sigma_{\pi}$ we get the following result which is one of the main steps of our argument.

LEMMA 2. $\tau_{\pi}$ being as in Lemma 1 put $\sigma_{\pi}=\Delta \tau_{\pi}$. Then

$$
\gamma(z) \sigma_{\pi}=\chi_{\pi}(z) \sigma_{\pi}
$$

for every $z \in \Re$.

Now choose a linear function $\Lambda$ on $\mathfrak{h}$ such that $\chi_{\pi}(z)=\Lambda(\gamma(z))$ for $z \in \mathfrak{B}$. Let $\zeta$ be an indeterminate and $u$ an element in $\mathfrak{u}$. Since $\mathfrak{u}$ is abelian we can consider the polynomial

$$
\prod_{s \in W}\left(\zeta-u^{s}\right)
$$

It is clear that every coefficient of this polynomial lies in $J$ and therefore if $w$ is the order of $W$, there exist uniquely determined elements $z_{1}(u), \cdots, z_{w}(u)$ in 3 such that

$$
\prod_{s} \in W\left(\zeta-u^{s}\right)=\zeta^{w}+\gamma\left(z_{1}(u)\right) \zeta^{w-1}+\gamma\left(z_{2}(u)\right) \zeta^{w-2}+\cdots+\gamma\left(z_{w}(u)\right) \text {. }
$$

On replacing $\zeta$ by $u$ we immediately get the identity

$$
u^{w}+u^{w-1} \gamma\left(z_{1}(u)\right)+u^{w-2} \gamma\left(z_{2}(u)\right)+\cdots+\gamma\left(z_{w}(u)\right)=0
$$

in $\mathfrak{U}$ if we recall that $\mathfrak{U}$ is abelian. Now apply the left side to $\sigma_{\pi}$ and 
use Lemma 2. Then

$$
u^{w} \sigma_{\pi}+\chi_{\pi}\left(z_{1}(u)\right) u^{w-1} \sigma_{\pi}+\cdots+\chi_{\pi}\left(z_{w}(u)\right) \sigma_{x}=0 .
$$

But $\chi_{\pi}\left(z_{j}(u)\right)=\Lambda\left(\gamma\left(z_{j}(u)\right)\right), 1 \leqq j \leqq w$, and since $\Lambda$ is a homomorphism of $\mathfrak{U}$ into $C$ it is obvious that

$$
\prod_{s \in W}\left(\zeta-\Lambda\left(u^{s}\right)\right)=\zeta^{w}+\Lambda\left(\gamma\left(z_{1}(u)\right)\right) \zeta^{w-1}+\cdots+\Lambda\left(\gamma\left(z_{w}(u)\right)\right) .
$$

Therefore the above differential equation for $\sigma_{\pi}$ may be written in the form

$$
\prod_{s \in W}\left(u-\Lambda\left(u^{s}\right)\right) \sigma_{\pi}=0 .
$$

However if $H_{1}, \cdots, H_{l}$ is a base for $\mathfrak{h}_{0}$ over $R$ and $\square=H_{1}^{2}+\cdots$ $+H_{l}^{2}$ it is obvious that the differential equation

$$
\prod_{s \in W}\left(\square-\Lambda\left(\square^{s}\right)\right) \sigma_{\pi}=0
$$

is of the elliptic type (see Garding [1]). Hence it follows from the work of Schwartz [4, p. 137] and John [3a, 3b] that $\sigma_{\pi}$ must be an analytic function on $A^{\prime}$. Now if we take into account Theorem 5 of [2b] and the fact that $\prod_{s \in W}\left(u-\Lambda\left(u^{s}\right)\right) \sigma_{\pi}=0$ for every $u \in \mathfrak{U}$, we get Theorem 2 without difficulty.

$\pi$ being any quasi-simple irreducible representation we denote by $T_{\pi}, \eta_{\pi}$, and $\chi_{\pi}$ respectively the character, the central character, and the infinitesimal character of $\pi$. Also we denote by $F_{\pi}$ the analytic function on $G^{\prime}$ such that $T_{\pi}=F_{x}$ on $G^{\prime}$. Let $T$ be a distribution on $G$. Since $D$ is an analytic function on $G$ the product $D^{m} T(m \geqq 0)$ is a well-defined distribution. It is then possible to prove the following result.

LEMмA 3. There exists an integer $m \geqq 0$ with the following property. Suppose $\pi_{1}, \cdots, \pi_{k}$ is a finite set of quasi-simple irreducible representations and $c_{1} F_{\pi_{1}}+\cdots+c_{k} F_{\pi_{k}}=0\left(c_{i} \in C\right)$. Then if $T=c_{1} T_{\pi_{1}}+\cdots$ $+c_{k} T_{\pi_{k}}, D^{m} T=0$.

From this lemma one can deduce the following theorem.

THEOREM 3. Let $\pi_{0}$ be a quasi-simple irreducible representation of $G$ such that $F_{\pi_{0}}$ is not identically zero. Then, apart from infinitesimal equivalence [2d, p. 230], there exist only a finite number of quasi-simple irreducible representations $\pi$ such that $F_{\pi}=F_{\pi_{0}}$.

Let $\eta \neq 0$ and $\chi \neq 0$ respectively be given homomorphisms of $Z$ and $\$$ into $C$. Let $\omega$ denote the set of all quasi-simple irreducible repre- 
sentations $\pi$ of $G$ such that $\eta_{\pi}=\eta$ and $\chi_{\pi}=\chi$. As we have seen, it follows from Theorems 1 and 2 that the functions $F_{\pi}(\pi \in \omega)$ span a finite-dimensional vector space over $C$. Hence if $\omega$ is not empty, we can choose a finite set of elements $\pi_{1}, \cdots, \pi_{k}$ in $\omega$ such that $F_{\pi_{1}}, \cdots$, $F_{\pi_{k}}$ form a base for this vector space. Then if $\pi$ is any representation in $\omega, F_{\pi}=c_{1} F_{\pi_{1}}+\cdots+c_{k} F_{\pi_{k}}\left(c_{j} \in C\right)$. If one could conclude from this equation that $T_{\pi}=c_{1} T_{\pi_{1}}+\cdots+c_{k} T_{\pi_{k}}$ it would follow (see [2e, Theorem 6]) that $\pi$ is infinitesimally equivalent to some $\pi_{j}(1 \leqq j \leqq k)$ and therefore, apart from infinitesimal equivalence, $\omega$ has only a finite number of representations. Therefore it is important to consider the following question.

Let $\pi_{1}, \cdots, \pi_{k}$ be a finite set of quasi-simple irreducible representations of $G$ such that $c_{1} F_{\pi_{1}}+\cdots+c_{k} F_{\pi_{k}}=0\left(c_{i} \in C\right)$. Then is it always true that $c_{1} T_{x_{1}}+\cdots+c_{k} T_{x_{k}}=0$ ?

I believe the answer is yes but do not know how to prove it.

\section{REFERENCES}

1. L. Gårding, Math. Scand. vol. 1 (1953) pp. 55-72.

2a. Harish-Chandra, Ann. of Math. vol. 50 (1949) pp. 900-915.

2b. - , Trans. Amer. Math. Soc. vol. 70 (1951) pp. 28-96.

2c. - Proc. Nat. Acad. Sci. U.S.A. vol. 37 (1951) pp. 366-369.

2d. - Trans. Amer. Math. Soc. vol. 75 (1953) pp. 185-243.

2e. - - Trans. Amer. Math. Soc. vol. 76 (1954) pp. 234-253.

2f. - Trans. Amer. Math. Soc. vol. 76 (1954) pp. 485-528.

3a. F. John, Comm. Pure Appl. Math. vol. 3 (1950) pp. 273-304.

3b. - Proceedings of the symposium on spectral theory and differential problems, Stillwater, Okla., 1951, pp. 113-175.

4. L. Schwartz, Théorie des distributions I, Paris, Hermann, 1950.

5. I. E. Segal, Proc. Amer. Math. Soc. vol. 3 (1952) pp. 13-15.

6. H. Weyl, Math. Zeit. vol. 24 (1925) pp. 328-395.

7. I. M. Gelfand and M. A. Naimark, Trudi Mat. Inst. Steklova vol. 36 (1950)

Columbia University 